\title{
Optimization of Logistics Network Configuration for Fresh Agricultural Products
}

\author{
Yingfeng $\mathrm{Ji}^{\mathrm{a}}$, Hualong Yang ${ }^{\mathrm{b}}$ and Yong Zhou ${ }^{\mathrm{c}}$ \\ School of Transportation Management, Dalian Maritime University, \\ Dalian, 116026, China \\ aJiyingfeng@lnmsa.gov.cn, ${ }^{b}$ hlyang@dlmu.edu.cn, ${ }^{c}$ z6a@163.com
}

\begin{abstract}
.
In order to optimize the logistics networks configuration of fresh agricultural products, the minimum cost related to the process, in which goods are delivered from original points, pre-cooling station, distribution center to demand points, was taken as the object function. By collecting the empirical decay index, and considering the time-sensitive features of fresh agricultural products, this paper established a nonlinear programming model and designed its corresponding algorithm. A case study was conducted based on genetic algorithm and the result shows validity of the model and effectiveness of its algorithm.
\end{abstract}

Keywords: logistics network; configuration; fresh agricultural product; optimization model; genetic algorithm

\section{Introduction}

Fresh agricultural products have the special feature of being easily depraved and rotted, the logistics network should be well designed with cold stores and refrigerated vehicles to keep quality and reduce waste and cost. As we know that the logistics cost is the main cost in food supply chain. Therefore, it's a hot topic of optimizing the fresh agricultural product supply chain network both in academic field and practical world. Vedat Verter (2002) discussed the location problem of multi-items in the production and distribution network [1]. Ruiz-Garcia Luis (2008) applied wireless sensor networks (WSN) for real-time monitoring of perishable fruits and vegetables logistics in refrigerated chambers and during transport, and established the model to estimate the water evaporation loss and detection of condensation [2]. Victoria Rodriguez (2011) verified the effectiveness of $6 \sigma$ as a methodological tool to adjust the equipment in the cold chain, and studied the impact of reducing the volume of storage in the truck, the initial temperature of the storage area in the truck and the frequency of defrost in the transport of refrigerated products [3]. Babalola, A.O. (2011) examined the cold chain logistics in the floral industry, and gave an extensive review of how flowers are transported around the world and concentrates on the Middle East [4]. Prakash G. (2012) focused on monitoring and adjusting the temperature in warehousing by using the combination of RFID Readers and Thermochron iButtons [5]. Carlos Renato Meneghetti (2013) presented the proposal and 
validation of a mathematical model for the cooling system of a cold-storage unit [6]. Cecilia (2014) used air temperature to predict the temperature of fresh fruits and vegetables for highest shelf-life prediction accuracy in the absence of individual sensors [7].

Though the above researches have enhanced the production capacity, the capacity constraints and flow constraints of alternative distribution center, and the time-sensitive features of fresh agricultural products, they have not taken the process time to deal with fresh agricultural products for pre-cooling station and distribution center. This could increase the decay cost of fresh agricultural products and then could not reach the goal of optimizing the logistics network. Therefore, this paper calculates the loss of logistics costs due to decay and established a nonlinear programming model for the logistics networks configuration of fresh agricultural products. And then it designs a genetic algorithm to solve the problem.

\section{Model}

In order to achieve optimization objectives of logistics network configuration of fresh agricultural products, the following issues need to be addressed: the location and number of pre-cooling stations and distribution centers, the transportation volume from distribution centers to points of demand. In general, this paper proposes the following basic assumptions:

Assuming that the existing distribution center can handle the fresh agricultural products, the distribution center location problem could be turned into some options for the selection of fresh agricultural product distribution center. The daily supply of origin which is not directly transported to the perishable distribution centers is in a small quantity, and each of the main production part including the retail and manufacturing base has a different supply rate that is the average daily shipments, which is called the average daily volume of purchases from the perspective of pre-cooling station; the distances between most of fresh agricultural origin and the nearby pre-cooling stations are very short so that the short-distance transportation costs and time between them are not taken into account; the concentration procurement cycle of pre-cooling stations are 3 days; the demand or each demand point is fixed and known.

To facilitate the narrative, we introduce the following symbols: $C_{k}^{r}$ is daily rental costs of distribution center $k$; $C_{j}^{d}$ is unit processing costs of pre-cooling station $j ; q_{i}^{s}$ is the supply volume of origin $i ; d_{i j}$ is distance from the origin i to the pre-cooling station $j$; $a_{j k}$ is unit freight from pre-cooling station $\mathrm{j}$ to distribution center $k ; b_{w}$ is the demand of demand point $w ; f_{k w}$ is unit freight from distribution center $k$ to demand point $w ; F_{k w}$ is distance from distribution center $k$ to demand point $w ; g_{k}$ is holding costs of distribution center $k$; $T$ is transportation time; $e$ is conversion factor, if $T<T_{0}$, then, $e=0 ; T_{0}$ is best quality hold time; $\rho_{w}$ is 
decay index; $I$ is the amount of place of origin; $J$ is the amount of pre-cooling station; $K$ is the amount of distribution center; $W$ is the amount of demand; $L_{(i, j) \max }$ is the maximum distance from origin $i$ to the pre-cooling station $k$; $Q_{k}^{c}$ is maximum capacity of distribution center $k$; $Q_{j}^{d}$ is the maximum capacity of a procurement cycle of the pre-cooling station $j ; T_{j}^{d}$ is unit processing time of pre-cooling station $j$; $T_{k}^{c}$ is unit processing time of distribution center $k . Z_{k}$ is $0-1$ variable, if distribution center $k$ is selected, then $Z_{k}=1 ; Y_{j}$ is $0-1$ variable, if pre-cooling station $j$ is selected, then $Y_{j}=1 ; X_{i j}$ is the quantity of supply from the origin $i$ to the pre-cooling station $j ; B_{k w}$ is the quantity of supply from distribution center $k$ to demand point $w ; A_{j k}$ is the quantity of supply from pre-cooling station $j$ to distribution center $k$.

With objective function of minimum total cost of the procurement cycle, we establish the following non-linear programming model.

$$
\min f=\sum_{k=1}^{K} 3 \times C_{k}^{r} Z_{k}+3 \sum_{j=1}^{J} C_{j}^{d} \sum_{i=1}^{I} q_{i}^{s} Y_{j} X_{i j}+\sum_{j=1}^{J} \sum_{k=1}^{K} \sum_{w=1}^{W} Z_{k}\left[d_{j k} a_{j k} A_{j k}+g_{k} A_{j k}+F_{k w} f_{k w} B_{k w}\right]+e \sum_{w=1}^{W} \rho_{w} b_{w}
$$

$$
\text { s.t. }\left\{\begin{array}{l}
d_{i j} X_{i j} \leq L_{(i, j) \max } \\
\sum_{j=1}^{J} A_{j k} \leq Z_{k} Q_{k}^{c} \\
\sum_{i}^{s} q_{i}^{s} X_{i j}=\sum_{k} A_{j k} \\
\sum_{i=1}^{I} X_{i j} q_{i}^{s} \leq Y_{j} Q_{j}^{d} \\
\sum_{j=1}^{J} A_{j k}=\sum_{w=1}^{W} B_{k w}
\end{array}\right.
$$

In equation (1), $\sum_{k=1}^{K} 3 C_{k}^{r} Z_{k}$ represents rental cost in a procurement cycle of the distribution centers; $3 \sum_{j=1}^{J} C_{j}^{d} \sum_{i=1}^{I} q_{i}^{s} Y_{j} X_{i j}$ represents processing cost of pre-cooling stations; $\sum_{j=1}^{J} \sum_{k=1}^{K} \sum_{w=1}^{W} Z_{k}\left[d_{j k} a_{j k} A_{j k}+g_{k} A_{j k}+F_{k w} f_{k w} B_{k w}\right]$ represents total transportation 
costs and warehousing costs; $e \sum_{w=1}^{W} \rho_{w} b_{w}$ represents losses in transit of Perishable products. $\rho_{w}$ also represents time-varying degree of decay of fresh agricultural products, which is the percentage of spoiled agricultural products. It is a time-related function. The time includes in-transit transportation time and product processing time taking in various pre-cooling stations and distribution centers. For the latter, this paper turns different processing times into transit times in calculation. Equation (2) represents the distance from the origin point to the pre-cooling station does not exceed the maximum distance restrictions; Equation (3) represents the quantity of supply that distribution center k received from all the supply points does not exceed its capacity limit; Equation (4) represents inbound flow of pre-cooling station $j$ equals its outbound flow; Equation (5) represents the procurement quantity that pre-cooling station $j$ purchased from the origins does not exceed its processing capacity constraints; Equation (6) represents the total import quantity equals to the export quantity in distribution center $k$.

\section{Algorithm}

In the above logistics network configuration optimization model, the decision variables consist of 0-1 variables and transportation volume $A_{j k}$. In practice, if measurement unit of transportation volume $A_{j k}$ is very large. For example, the unit measurement of cars will be turned into the unit measurement of small container, thus the number will be translated into integer and the problem of the model will be integer nonlinear programming problem. Regarding to such a non-linear programming problems, we use rounded relaxation and branch and bound method such classical mathematics methods to solve them, but when there exiting too many network nodes, this problem turns out to be NP hard problem. There will be curse of dimensionality when using such methods, therefore, this paper will use genetic algorithms to solve the problem.

Since the model involves fresh pre-cooling station and distribution center site selection decision-making and traffic decision-making, this paper combines binary encoding and floating-point encoding methods to code decision variables of the model. $Y_{j}$ and $Z_{k}$ are 0-1 variables. Therefore, we use binary encoding method to encode, regarding to $X_{i j}, A_{j k}, B_{k w}$, because of high number of variables, therefore, floating-point number coding method should be adopted.

Logistics network configuration problem is a constrained optimization problem. The constraints in this mathematical model are in large scale and feasible solutions in the population take a considerable proportion, so the problem needs to be translated into unconstrained problems. Penalty function method is used to handle constraints. Regarding to those individuals without corresponding feasible solutions, when calculating its fitness, are punished by a penalty function, thereby 
reducing the fitness of the individual and the chances that those individuals are inherited to the next generation of the group are reduced.

According to the model constraints, let $t_{j}=L_{(i, j) \max }-d_{i j} X_{i j}$, $r_{k}=Z_{k} Q_{k}-\sum_{j=1}^{J} A_{j k} \quad, \quad q_{j}=Y_{j} Q_{j}^{d}-X_{i j} q_{i}^{s} \quad, \quad P_{j}=\left|\sum_{i} q_{i}^{s} X_{i j}-\sum_{k} A_{j k}\right|$, $w_{k}=\left|\sum_{j=1}^{J} A_{j k}-\sum_{w=1}^{W} B_{k w}\right|$. Based on the penalty function $P(X)=\frac{1}{2 t} \sum_{q \in A} d_{i}^{2}(X)$ proposed by Michalewicz and Attia, we introduce the simulated annealing algorithm(7) and design the punish factor, $\tau=\frac{\tau_{0}}{1+t}$, we obtain the following penalty function:

$$
P=\left\{\begin{array}{lc}
0 & \text { satisfies the constraint } \\
\frac{t+1}{2 \tau_{0}} \sum_{q=1}^{A} d_{q}^{2} & \text { otherwise }
\end{array}\right.
$$

Where $\mathrm{t}$ is hereditary algebra; $\tau_{0}$ is initial factor.

As we can see, in the early iterations of genetic algorithms, infeasible solutions are little punished to limit the algorithm's premature convergence, while in the latter part of the iteration, the method will severe punish the infeasible solutions in order to avoid random search.

Therefore, the fitness function can be defined as:

$F^{\prime}(X)=\left\{\begin{array}{lc}F(X) & \text { When } X \text { satisfies the constraint } \\ F(X)-P(X) & \text { otherwise }\end{array}\right.$

In equation (8): $F(X)$ is the original fitness; $F(X)$ means the new fitness after considering the penalty function; $F(X)$ is penalty function. Decoding and terminate the Algorithm.

We use the optimum selection method to ensure that the best chromosome in the case of not being selected will appear in the next generation and the number of initial population is 25 . We adopt random selection method, and the crossover method is two-points intersection, while mutation probability is 0.05 . If the continuous generation of 100 is not improved, then the operation will be terminated.

The specific calculation process of genetic algorithm designed in this paper is shown in Fig.1: 


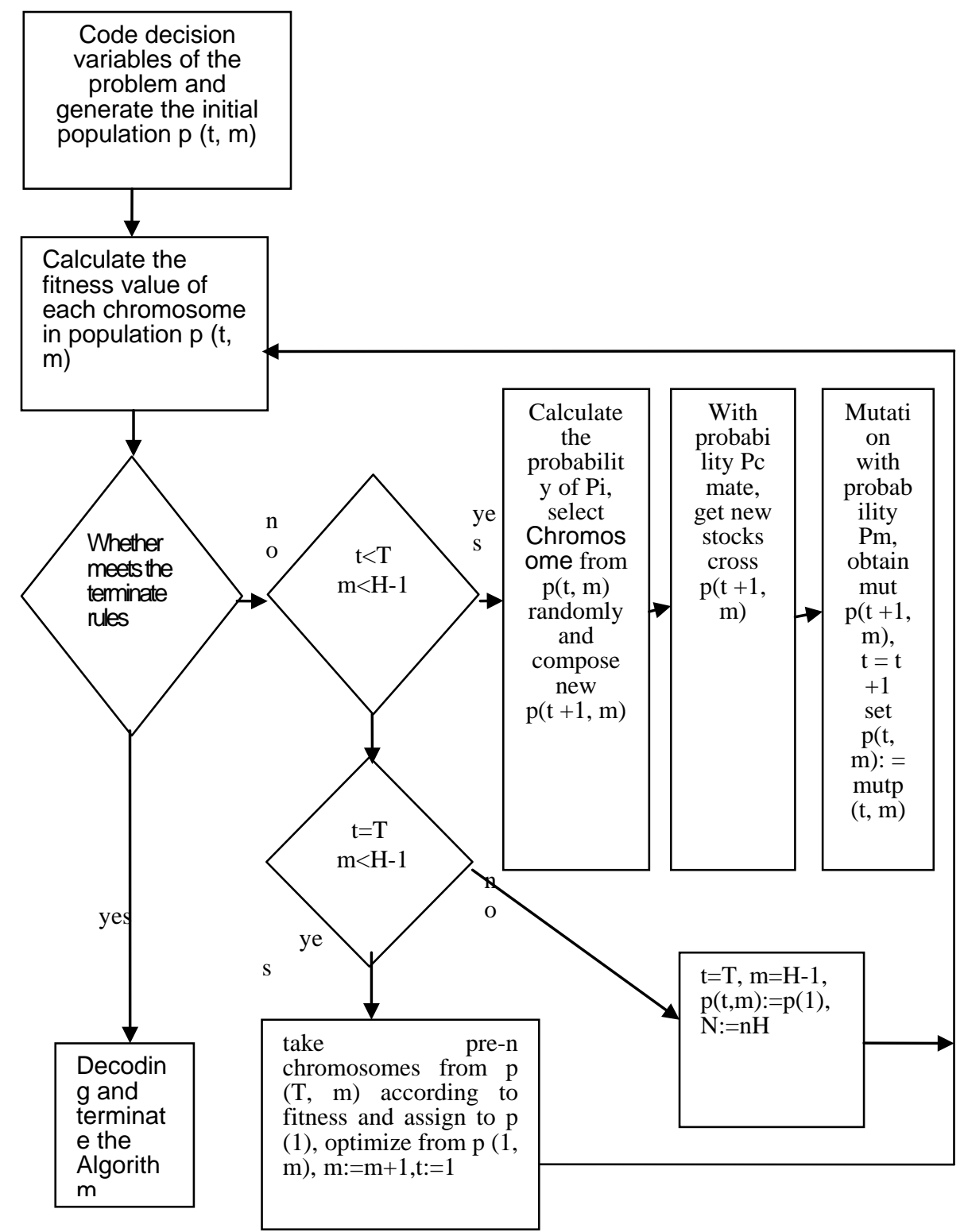

Fig. 1 Genetic algorithm flow chart

\section{Empirical Analysis}

Suppose there are six strawberry production bases, four alternative pre-cooling stations, three alternative distribution centers, nine demand points. The maximum 
number of restrictions of pre-cooling stations and distribution centers are 4 and 2 . The maximum capacity of the procurement cycle of a pre-cooling station is 160 . In this case, the storage temperature of strawberry from picking points to demand points is $4^{\circ} \mathrm{C}$ and the radiation dose is $1.0 \mathrm{kgy}$. We get the function of degree of strawberry decay function with the number of days as follows:

$$
y=0.0443\left(\frac{T_{w}-3}{3}\right)^{2}-0.0237\left(\frac{T_{w}-3}{3}\right)+0.024, R^{2}=0.9972 .
$$

In this paper, we use genetic algorithm-assisted auxiliary Toolbox matlab7.0 to solve the problem. Based on the parameters set in the algorithm design, we run the problem several times in the toolbox when we achieve the optimal solution. The average run algebra is 85 generations. Finally, we get the approximate optimal solution in this case that is the network nodes of the optimal layout making a smallest total cost of in a procurement cycle. Select the pre-cooling station 2 and 3 , distribution centers 1 and 3 . Among them, pre-cooling station 2 is responsible for procurement of the origin of 1,2,3, through the distribution center 1 to dispatch the needs of points 1,7,8,9; Pre-cooling station 3 is responsible for the purchase of the rest of the origin, through the distribution center 3 to ration the remaining demand points.

The distribution result is shown in Fig.2:

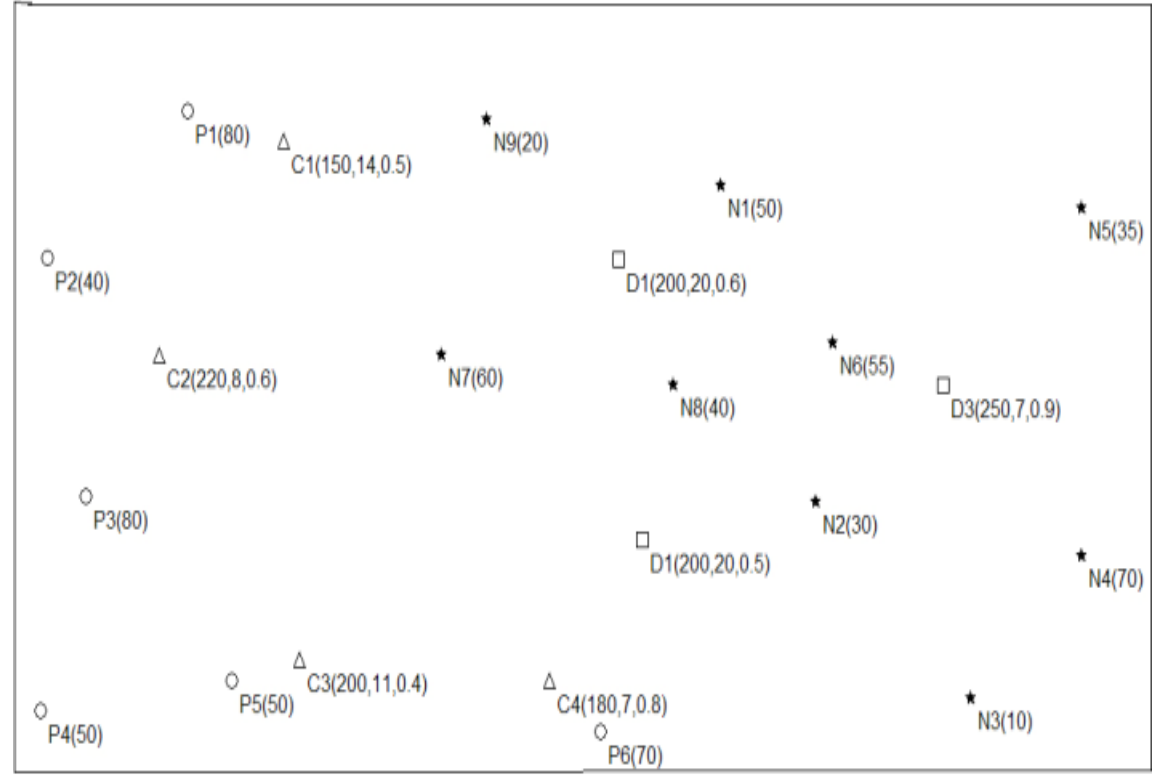

Note: ○— Origin $\left(q_{i}^{s}\right) ; \triangle$-Pre-cooling station $\left(Q_{j}^{d}, C_{j}^{d}, T_{j}^{d}\right)$; $\square$ —Distribution

$$
\text { Center }\left(Q_{k}^{c}, C_{k}^{r}, T_{k}^{c}\right) ; \star \text { Demand Point }\left(b_{w}\right) \text {. }
$$

Fig.2. Distribution result.

\section{Conclusions}


This paper calculates the loss of logistics costs due to decay and established a nonlinear programming model for the logistics networks configuration of fresh agricultural products. And then it designs a genetic algorithm to solve the problem. The result of case study shows the validity of the model and effectiveness of its algorithm.

\section{References}

[1] Sirisak K. (2005) Shelter Location-allocation Model for Flood Evacuation Planning. Journal of the Eastern Asia Society for Transportation Studies, 6, 4237-4252.

[2] Ruiz-Garcia L, Barreiro, P., Anand, A., Robla, J.I. (2008) Modelling Psychometric Data in Real-time Fruit Logistics Monitoring. 4th International Symposium on Applications of Modelling as an Innovative Technology in the Agro-Food-Chain-Model-IT, Madrid, Spain, 385-390.

[3] Victoria R, Itxaso, A., Maria, J.A. (2011) Setting Parameters in the Cold Chain. Tecnura, 15(30), 71-81.

[4] Babalola, A.O., Sundarakani, B.; Ganesh, K. (2011) Cold Chain Logistics in the Floral Industry. International Journal of Enterprise Network Management, 4(4), 400-413.

[5] Prakash G., Pravin, R., Venkatalakshmi, B. (2012) RFID based Mobile Cold Chain Management System for Warehousing International Conference on Modelling Optimization and Computing, 38, 964-969.

[6] Carlos R M, Alexandre, T., Nelson, L.C., Claudio, K.U., Guilherme, B. (2013) A Mathematical Model for the Cold Storage of Agricultural Products. Agricultural Engineering Scientific Papers, 44(2), 286-293.

[7] Cecilia, D.N., Mike N., Jean, P.E. (2014) Improvement in Fresh Fruit and Vegetable Logistics Quality: Berry Logistics Field Studies. Philosophical Transactions of the Royal Society A-mathematical Physical and Engineering Sciences, 372(2017), 13-17. 